\title{
Retrieval of Dislodged Coronary Intravascular Ultra- sound Catheter With Embolic Protection Device
}

\author{
Chih-Ping Chang, ${ }^{1}$ MD, Jen-Jyh Lin,,${ }^{1}$ MD, Jui-Sung Hung, ${ }^{1}$ MD, \\ Pei-Ying PAI, ${ }^{1} \mathrm{MD}$, and Chung-Ho Hsu, ${ }^{1} \mathrm{MD}$
}

\section{SUMMARY}

A retained fractured segment of an intravascular ultrasound catheter in the coronary artery during percutaneous coronary intervention is a rare occurrence. We describe our experience with successful retrieval of a fractured IVUS catheter fragment in a previously stented left anterior descending artery using a distal embolic protection device. (Int Heart J 2009; 50: 121-125)

Key words: Percutaneous coronary intervention, Retrieval technique

LOST and retained device components in the coronary arteries during percutaneous coronary intervention include stents, fragmented guidewires, and the tips of balloon catheters. ${ }^{1)}$ To the best of our knowledge, the fracture of an intravascular ultrasound (IVUS) catheter in the coronary artery has seldom been reported in the English literature. ${ }^{2,3)}$ We report fracture and dislodgment of a distal IVUS catheter within a stent. After commonly used stent retrieval methods, including the use of a loop snare, double-wires, and a distally placed angioplasty balloon, ${ }^{1)}$ the retained distal catheter fragment was retrieved using a Filter Wire, which is a distal embolic protection device (EPD).

\section{Case Report}

Percutaneous coronary intervention (PCI) was performed to the left anterior descending (LAD) artery via the right femoral artery approach in an 82-year-old woman who presented with unstable angina. The proximal LAD artery segment was heavily calcified and showed severe stenosis on angiography (Figure 1A). There was also severe calcification in the proximal left circumflex artery segment. The right coronary artery was a nondominant artery without any

From the ${ }^{1}$ Division of Cardiology, Department of Internal Medicine, China Medical University Hospital, Taichung, Taiwan.

Address for correspondence: Jui-Sung Hung, MD, Division of Cardiology, Department of Internal Medicine, China Medical University Hospital, Yuh-Der Road, Taichung 404, Taiwan.

Received for publication February 21, 2008.

Revised and accepted October 16, 2008. 
significant stenosis. The LAD was cannulated with a 6F EBU 3.5 guiding catheter (Medtronic, Danvers, Minneapolis, USA), and a 0.014" ATW floppy wire (Cordis, Miami Lakes, Florida, USA) was anchored deep in the LAD artery. The lesion was dilated using a $3.0 \times 20 \mathrm{~mm}$ Sprinter RX catheter balloon (Medtronic, Galway, Ireland) and subsequently treated with a $3.0 \times 24 \mathrm{~mm}$ bare-metal Driver stent (Medtronic, Galway, Ireland) because of a suboptimal outcome after the former procedure. A dissection was suspected at the distal edge of the stent (Figure 1B), prompting further interrogation with the use of an Atlantis SR pro 40 MHZ Coronary Image IVUS system (Boston Scientific, Natick, Massachusetts, USA). However, the IVUS catheter tip could not be advanced beyond the distal stent segment. When the IVUS catheter was withdrawn, resistance was felt, and under fluoroscopy the distal tip separated with the IVUS catheter from the distal part of the stent. The fractured tip could be moved slightly toward the proximal portion of the stent (Figure 1C) before it went beyond the IVUS wire.

The following retrieval attempts failed: First, a $4 \mathrm{~mm}$ GooseNeck Amplatz Microsnare catheter (Microvena Corp., White Bear Lake, MN, USA) was passed over the guidewire and inserted through a $4 \mathrm{~F}$ transport catheter.
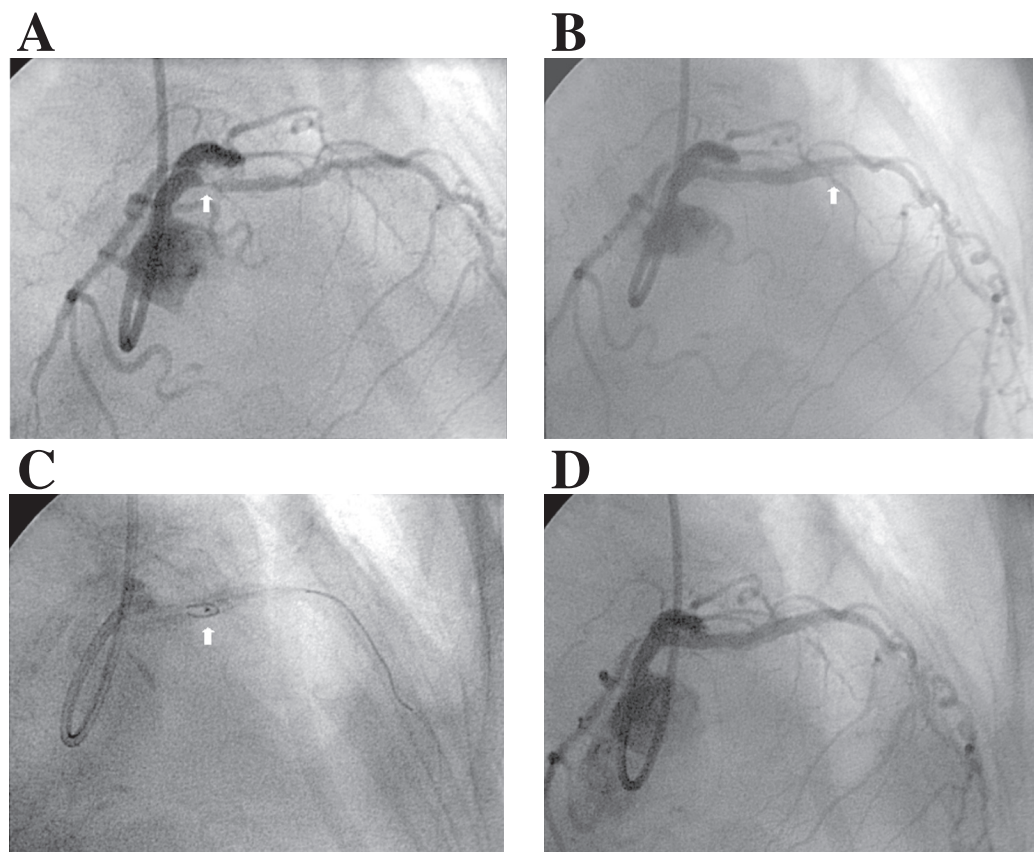

Figure 1. Angiograms in right anterior oblique and cranial view. A: a severe stenosis (white arrow) in proximal left anterior descending artery. B: After placement of a stent, dissection (white arrow) is suspected just distal to the stent. C: IVUS segment is retained in Filter Wire basket. White arrow indicates metal band in the distal IVUS catheter segment contained in the Filter Wire basket metal ring. D: Final coronary angiogram. 

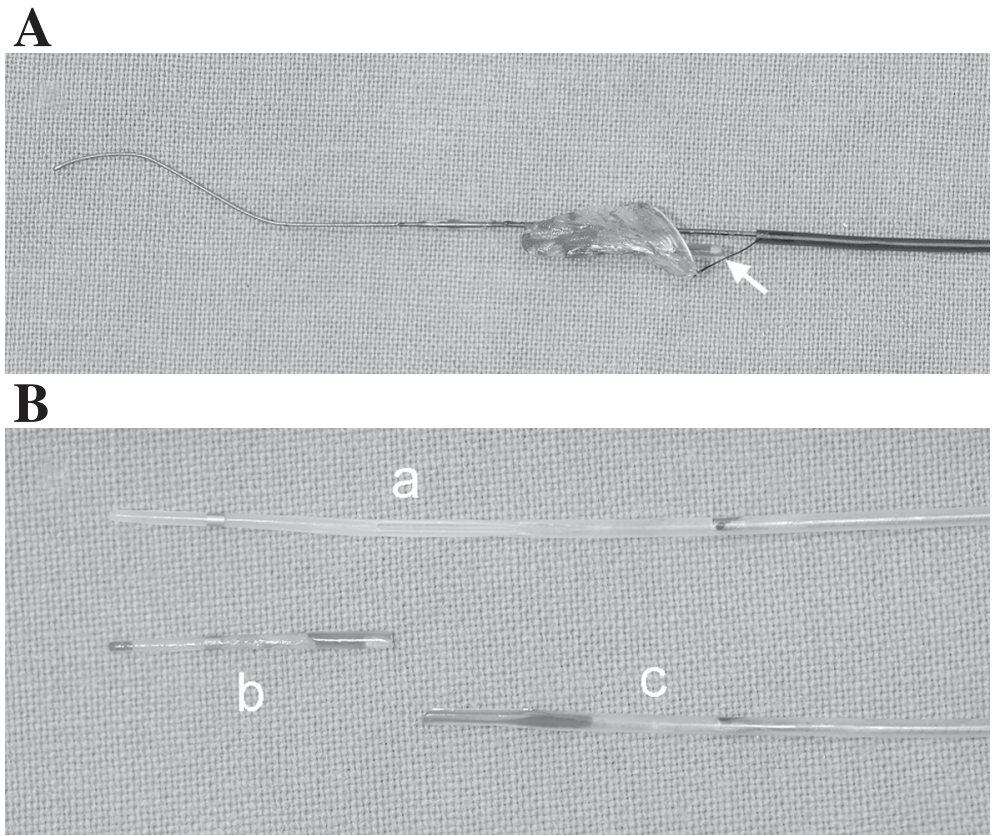

Figure 2. A: Retrieved fractured IVUS catheter segment (white arrow) in FilterWire basket. B: Intact IVUS catheter (a), fractured distal IVUS catheter (b) (about $15 \mathrm{~mm}$ in length) and proximal IVUS catheter segment (c).

However, it failed to snare the IVUS catheter fragment. Second, a Fielder wire (ASAHI, NEO's, Japan) was placed deeply in the LAD as a buddy wire, and both ends of the guidewires were fixed in a torque device. Despite rotating the torque, the distal ends of the 2 wires could not be entwined to enable retrieval of the retained fragment. Therefore, the first wire threading the IVUS catheter was removed. Lastly, a $2.5 \times 12 \mathrm{~mm}$ Sprinter RX balloon catheter (Medtronic, Galway, Ireland) was inserted beyond the stent segment over the second wire. As the catheter was withdrawn, the balloon was inflated to act as a distal retainer to retrieve the IVUS catheter. However, multiple attempts using this method were also unsuccessful in retrieving the IVUS catheter.

After the above 3 methods failed, a FilterWire EZ (Boston Scientific, Natick, Massachusetts) was inserted through a transport catheter beyond the stent. The Filterwire was withdrawn, and after having confirmed containment of the IVUS catheter fragment within the basket (Figure 1C), the FilterWire together with the transport catheter and IVUS catheter fragment were retrieved inside the guiding catheter and exteriorized (Figure 2B). A second $3.0 \times 24 \mathrm{~mm}$ Driver stent (Medtronic, Galway, Ireland) was then deployed to cover the distal stent edge dissection (Figure 1D). During the entire PCI procedure, the patient re- 
mained asymptomatic and was hemodynamically stable.

\section{Discussion}

Lost and retained device components in the coronary arteries during PCI include stents, fragmented guidewires, and the tips of balloon catheters. ${ }^{1)}$ While coronary stents are the most commonly lost device, the frequency of stent loss has decreased from $1.04 \%$ to $0.27 \%$ since the use of factory-premounted stents. ${ }^{4)}$ We encountered the unusual occurrence of a fractured IVUS catheter lodged in the preplaced stent in the proximal LAD artery. The common mechanism of IVUS entrapment after coronary artery stenting includes malapposed stent struts, catheter deformation from multiple uses, and loss of wire position. In our patient, the fractured IVUS possibly resulted from a small portion of the stent strut jutting out slightly into the lumen due to the calcified vessel and the occurrence of the entrapment between the guidewire and stent strut at the site of the guidewire exit port. High-pressure balloon inflations of the stent or an extrasupport or extra-stiff wire may prevent the dislodged IVUS.

The most ideal solution to retain a device is percutaneous extraction with a snare. Insertion of a second stent to compress the lost device is the second choice, while surgical extraction is the third. Retrieval of the dislodged IVUS fragment was unsuccessful in our patient by commonly used methods of stent retrieval, including the use of a loop snare, twisted double-wires, and a distally placed angioplasty balloon. ${ }^{1)}$ Finally, the retained IVUS catheter fragment was retrieved using a FilterWire meant for distal embolic protection. We resolved this problem in a very short time even though the EPD is not preinserted and the dislodged IVUS catheter is not coaxial with the EPD. The use of a distal embolic protection device has been reported in retrieving lost stents in aorto-coronary saphenous vein grafts. ${ }^{5,6)}$ Webb, et al $^{5)}$ used a preplaced 0.014" GuardWire (PercuSurge, Sunnyvale, CA) device to retrieve a lost stent in a saphenous graft to the circumflex artery. Guigauri, et $a l^{6}{ }^{6}$ utilized a preplaced FilterWire (Boston Scientific, Natick, Massachusetts) distal embolic protection device to retrieve a lost stent in the distal vein graft to the obtuse marginal artery after having failed to do so with a GooseNeck snare and a second angioplasty balloon.

In this report, the first of its kind, we offer an alternative and practical method, namely the use of the FilterWire, in retrieving retained IVUS catheter fragments and possibly other devices, within the coronary circulation during PCI. 


\section{REFERENCES}

1. Brilakis ES and Garratt KN. Device loss during percutaneous coronary intervention: Incidence, complication, and retrieval methods. In: Stephen G. Ellis, MD., David R. Holmes, Jr., MD. Strategic approaches in coronary intervention. Philadelphia: Lippincott Williams and Wilkins Inc; 2006. P434-44.

2. Batkoff BW, Linker DT. Safety of intracoronary ultrasound: data from a Multicenter European Registry. Cathet Cardiovasc Diagn 1996; 38: 238-41.

3. Sasseen BM, Burke JA, Shah R, et al. Intravascular ultrasound catheter entrapment after coronary artery stenting.Catheter Cardiovasc Interv 2002; 57: 229-33.

4. Eggebrecht H, Haude M, von Birgelen C, et al. Nonsurgical retrieval of embolized coronary stents. Catheter Cardiovasc Interv 2000; 51: 432-40.

5. Webb JG, Solankhi N, Carere RG. Facilitation of stent retention and retrieval with an emboli containment device. Cathet Cardiovasc Interv 2000; 50: 215-7.

6. Guigauri P, Dauerman HL. A novel use for a distal embolic protection device: stent retrieval. J Invasive Cardiol 2005; 17: 183-4. 